\title{
THE VALIDITY OF THE HAMILTON DEPRESSION RATING SCALE AS A SCREENING AND DIAGNOSTIC INSTRUMENT FOR DEPRESSION IN PATIENTS WITH EPILEPSY
}

\author{
Koraliya S. Todorova ${ }^{1}$, Valentina S. Velikova ${ }^{2}$ \\ 1) First Clinic of Psychiatry, MHAT "Sveta Marina"-Varna, Bulgaria \\ 2) Faculty of Dental Medicine, Medical University-Varna, Bulgaria
}

\begin{abstract}
Aim: To evaluate the concurrent validity of the Hamilton Depression Rating Scale (HAMD-17) against ICD10 criteria for depressive disorder and its performance as a screening and diagnostic tool for depression in patients with epilepsy (PWE).

Subjects and Methods: One hundred and six PWE underwent clinical psychiatric examination followed by evaluation on HAMD-17. ICD-10 criteria for comorbid depressive disorder were applied. Internal consistency was assessed using Cronbach's $\alpha$. A "receiver operating characteristics" (ROC) curve was obtained and the sensitivity, specificity, positive and negative predictive values (PPV, NPV) were calculated for different cut-off points of the HAMD-17.

Results: Internal consistency measured by Cronbach's \pm was 0.74 . Maximal discrimination between depressed and non depressed was obtained at a cut-off score of $8 / 9$ (sensitivity 0.93 , specificity 0.98 ). High sensitivity and NPV at the same cut-off score (sensitivity 0.93, NPV 1.0) show the screening properties, and high specificity and PPV at cut-offs $9 / 10$, the diagnostic properties of the instrument. The area under the ROC curve $(\mathrm{AUC}=0.746)$ indicates the concurrent validity of the HAMD-17 score with the ICD-10 criteria for depressive disorder.

Conclusion: The validity of the HAMD-17 against ICD-10 criteria for depressive disorder in PWE in our study is fair. The concurrent administration of diagnostic criteria can ascertain the presence of core symptoms of depression.
\end{abstract} Epilepsy

Key words: Psychometric properties, HAMD-17,

\section{INTRODUCTION:}

The 17-item Hamilton Depression Rating Scale (HAMD-17) is one of the most widely used instruments in depression assessment and research (1). Although originally constructed as an interviewer-rated instrument for evaluation of the severity of depression without significant somatic or organic brain comorbidity, semistructured versions are often used in clinical practice for different patient populations in which their validity is to be established(2). In the last decade the psychometric properties of HAMD-17 in patients with stroke, Alzheimer's dementia and Parkinson's disease and its concurrent validity with DSM-IV criteria for major depressive disorder in these patient groups have been investigated $(2,3)$. For optimal performance different cut-off points have been taken into consideration for each organic disorder $(3,4)$.

Whereas depressive disorders are the most frequent psychiatric comorbidity in epilepsy with a significant negative impact on the quality of life of the patients (5), aim of our study was to evaluate the concurrent validity of the HAMD17 in relation to the ICD-10 criteria for depressive disorder and to assess its use as a screening and diagnostic tool for depression in patients with epilepsy (PWE).

\section{SUBJECTS AND METHODS:}

One hundred and six patients (41males, 65 females) aged 18-60 years with a confirmed diagnosis of partial epilepsy or of primarily generalized epilepsy $(6,7)$ with a minimal duration of 12 months after its confirmation by a neurologist, without any other somatic or neurological comorbidity were included in the study. All patients underwent clinical psychiatric examination including evaluation on HAMD-17. ICD-10 diagnostic criteria for depressive disorder were followed. All interviews and scales were administered by trained staff. Patients with other than depression psychiatric comorbidity were excluded from the analysis.

Internal consistency was assessed using Cronbach's $\alpha$. A "receiver operating characteristics" (ROC) curve was obtained and the sensitivity, specificity, positive and negative predictive values (PPV, NPV) were calculated for different cut-off points of the HAMD-17, illustrating the discriminative properties of the scale in the selected patient population. Data processing and analysis were carried out using SPSS for Windows (Version 15.0).

\section{RESULTS:}

The average age of the patients in the study was 37.16 \pm 11.57 . Thirty patients met the ICD-10 criteria for depressive 
disorder, corresponding to a prevalence of $28.3 \%$. Female gender prevailed over male, respectively $20(66.7 \%)$ and $10(33.3 \%)$ of all depressed. Mean HAMD-17 scores, ranges and standard deviations are listed in Table 1. for the depressed and non-depressed subgroups. Cronbach's $\alpha$ was 0.74 , proving the internal consistency of the scale. Sensitivity, specificity, positive and negative predictive values for different cut-off scores are shown in Table 2. Maximal discrimination between depressed and non-depressed was obtained at a cut-off score of $8 / 9$ (sensitivity 0.93 , specificity 0.98 ), meaning that a score of 8 or less indicates the absence of depression and a score of 9 or higher is indicative for the presence of depression. For screening purposes high sensitivity and NPV are required and were obtained at the same cut-off score (sensitivity 0.93, NPV 1.0). At higher cutoffs $-9 / 10$, the high specificity and PPV show the diagnostic properties of the scale in PWE. Figure 1 displays the results in the form of a ROC curve. The $\mathrm{AUC}=0.746$ indicates that the concurrent validity of the HAMD-17 score with the ICD10 criteria for depressive disorder in our study is fair.

\section{DISCUSSION:}

As the interviewer-rated HAM-D-17 is the most widely used and accepted measure for evaluating the severity of depression (8), we determined the concurrent validity of the HAMD-17 with ICD-10 criteria for depressive disorder in PWE. However optimal performance requires that different cut-off points should be taken into consideration for different organic disorders e.g. stroke, Parkinson's disease, Alzheimer's dementia, epilepsy (3). The prevalence of depression in our sample was within the range reported by other authors $(9$, $10,11,12)$. Our results show that at low cut-off scores HAMD-17 can be used as a screening instrument for depression in epilepsy. Requirements that a screening instrument should be quick and easy to administer give self-report questionnaires an obvious advantage over interviewer rating scales, especially in routine clinical work or in large-scale epidemiological studies as they are less work intensive and do not need trained personnel to administer them $(3,4)$. Overall the better psychometric properties of the observer-rated scales compared to self-rated, make them preferable if the study or clinical situation permits (4). Yet training in the administration and scoring of the scale is important to obtain reliable scores (13).

Although at higher cut-off scores of 9/10 HAMD-17 functioned well as a diagnostic instrument for depression in PWE the diagnosis should not be solely made on the basis of a score on a rating scale. We share the opinion that a cutoff score on an instrument cannot comprehensively capture the range of depressive disorders in epilepsy (3); high scores can occur when somatic symptoms are endorsed even without the two core symptoms of depression (i.e. sad mood and loss of interest or pleasure); low scores can occur despite serious depressive symptoms when somatic or vegetative symptoms are absent. This makes the reason why the gold standard for establishing the diagnosis of depression remains an interview using ICD-10 respectively DSM-IV criteria or their equivalent future diagnostic adaptations.

\section{CONCLUSION:}

The validity of the HAMD-17 against ICD-10 criteria for depressive disorder in PWE in our study is fair. The clinical practice to use the scale to measure depressive symptoms in both non-depressed and depressed epilepsy patients and to dichotomize patient samples on the basis of depression is justified. The concurrent administration of diagnostic criteria can ascertain the presence of core symptoms of depression.

Table 1. Mean HAMD-17 scores in patients with epilepsy with and without comorbid depressive disorder

\begin{tabular}{|c|c|}
\hline \multicolumn{2}{|c|}{ Mean \pm SD (Range) } \\
\hline Nondepressed & Depressed \\
\hline $1,84 \pm 2,00(0-7)$ & $13,76 \pm 5,62(8-30)$ \\
\hline
\end{tabular}

Table 2. Sensitivity, specificity, positive and negative predictive values at different cut-off scores for the HAMD-17 in patients with epilepsy

\begin{tabular}{|l|c|c|c|c|c|c|c|c|c|c|}
\hline Cut-off & $\mathbf{7 / 8}$ & $\mathbf{8 / 9 *}$ & $\mathbf{9 / 1 0}$ & $\mathbf{1 0} / \mathbf{1 1}$ & $\mathbf{1 1 / 1 2}$ & $\mathbf{1 2 / 1 3}$ & $\mathbf{1 3 / 1 4}$ & $\mathbf{1 4 / 1 5}$ & $\mathbf{1 5 / 1 6}$ & $\mathbf{1 6 / 1 7}$ \\
\hline Sensitivity & 0.92 & 0.93 & 0.89 & 1 & 0.92 & 0.72 & 1 & 1 & 0.6 & 0.58 \\
\hline Specificity & 0.68 & 0.98 & 0.98 & 0.71 & 0.72 & 1 & 0.89 & 0.86 & 0.83 & 0.94 \\
\hline PPV & 0.53 & 0.76 & 0.86 & 0.73 & 1 & 0.65 & 0.7 & 0.76 & 0.83 & 0.64 \\
\hline NPV & 0.78 & 1 & 0.92 & 0.75 & 0.75 & 0.87 & 0.86 & 0.83 & 0.79 & 0.76 \\
\hline
\end{tabular}


Fig.1. Receiver operating characteristic cuvre for the HAMD-17 in patients with epilepsy

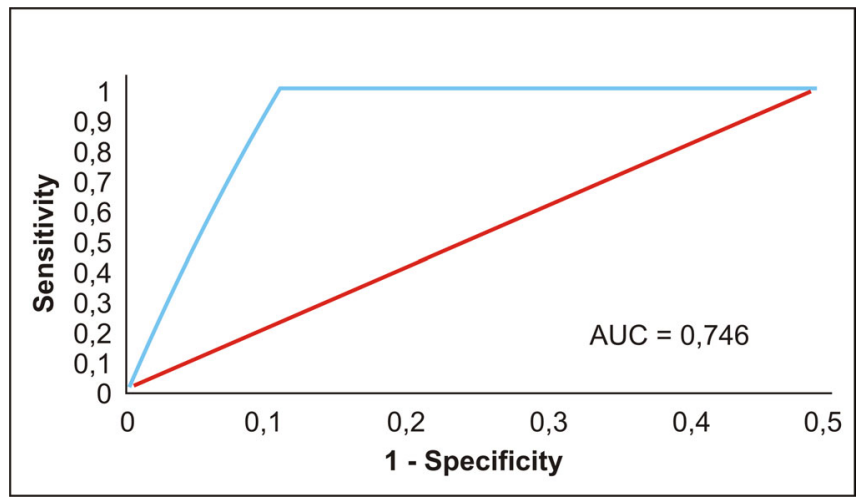

$\mathrm{AUC}=$ area under the curve

\section{REFERENCES:}

1. Hamilton M. A rating scale for depression. $J$ Neurol Neurosurg Psychiatry. 1960 Feb;23:56-62. [PubMed]

2. Leentjens AF, Verhey FR, Lousberg R, Spitsbergen H, Wilmink FW. The validity of the Hamilton and Montgomery-Asberg depression rating scales as screening and diagnostic tools for depression in Parkinson's disease. Int J Geriatr Psychiatry. 2000 Jul;15(7):644-649. [PubMed] [CrossRef]

3. Naarding P, Leentjens AF, van Kooten F, Verhey FR. Disease-specific properties of the Hamilton Rating Scale for Depression in patients with stroke, Alzheimer's dementia, and Parkinson's disease. $J$ Neuropsychiatry Clin Neurosci. 2002 Summer;14(3):329-334. [PubMed] [CrossRef]

4. Schrag A, Barone P, Brown RG, Leentjens AF, McDonald WM, Starkstein S, et al. Depression Rating Scales in Parkinson's Disease: Critique and Recommendations. Mov Disord. 2007 Jun 15;22(8):1077-1092. [PubMed] [CrossRef]

5. Kanner AM, Wuu J, Barry J, Hermann B, Meador KJ, Gilliam F.
Atypical depressive episodes in epilepsy: a study of their clinical characteristics and impact on quality of life. Neurology. 2004; 62(supp15):A249.

6. Proposal for revised clinical and electroencephalographic classification of epileptic seizures. From the Commission on Classification and Terminology of the International League Against Epilepsy. [No authors listed]. Epilepsia. 1981 Aug;22(4):489-501. [PubMed]

7. Proposal for revised classification of epilepsies and epileptic syndromes. Commission on Classification and Terminology of the International League Against Epilepsy. [No authors listed]. Epilepsia. 1989 Jul-Aug;30(4):389-399. [PubMed]

8. Guy W. ECDEU assessment manual for psychopharmacology. Rockville, Md.: U.S. Dept. of Health, Education, and Welfare, Public Health Service, Alcohol, Drug Abuse, and Mental Health Administration, National Institute of Mental Health, Psychopharmacology Research Branch, Division of Extramural Research
Programs. 1976; p217-222. [Full Text]

9. Kanner A, Balabanov A. Depression and epilepsy: how closely related are they? Neurology. 2002 Apr 28;58(8 Suppl. 5):S27-S39. [PubMed]

10. Tellez-Zenteno JF, Patten SB, Jette N, Williams J, Wiebe S. Psychiatric comorbidity in epilepsy: a populationbased analysis. Epilepsia. 2007 Dec; 48(12):2336-2344. [PubMed] [CrossRef]

11. Titlic M, Basic S, Hajnek S, Lusic I. Comorbidity psychiatric disorders in epilepsy: a review of literature. Bratisl Lek Listy. 2009; 110(2):105-109. [PubMed]

12. Ettinger A, Reed M, Cramer J; Epilepsy Impact Project Group. Depression and comorbidity in communitybased patients with epilepsy or asthma. Neurology. 2004 Sep 28;63(6)1008-1014. [PubMed]

13. Muller MJ, Dragicevic A. Standardized rater training for the Hamilton Depression Rating Scale (HAMD-17) in psychiatric novices. $J$ Affect Disord. 2003 Oct;77(1):65-69. [PubMed] [CrossRef]
Address for correspondence:

Koraliya Todorova, MD, PhD

First Clinic of Psychiatry, MHAT "Sveta Marina"

1, Hristo Smirnenski str., 9010 Varna, Bulgaria

Mobile: +359 888376327

E-mail: koralia_todorova@yahoo.com; 